\title{
MAINTENANCE OF THE ICRS - STATISTICAL TREATMENT
}

\author{
H. SCHWAN \& R. WIELEN \\ Astronomisches Rechen-Institut \\ Moenchhofstrasse 12-14, D-69120 Heidelberg, Germany
}

\section{Introduction}

In this paper we briefly describe the influence of astrometric binaries on derived proper motions and we draw some conclusions with respect to the reference systems. For more details the reader is referred to Wielen (1997) and Wielen et al. (1997).

Increased measurement accuracy determines not only the derived parameters with much more precision, but it may even allow one to describe new phenomena, which were of no importance before. This happened in the case of the highly precise Hipparcos measurements (ESA, 1997) which were able to determine stellar proper motions within less than four years with an accuracy comparable with the best proper motions available from ground-based observations as given in the FK5 (Fricke et al. 1988). The basic difference between the Hipparcos and the FK5 proper motions showed up within our task of validating the Hipparcos proper motions during the reduction process. Our main result was that the dispersion of the residual differences between both proper motions was about three times as large compared with the error estimates expected from the data given in the catalogues. Since it was rather unlikely that one of the two catalogues had so strongly underestimated its errors, we looked for other reasons to explain the discrepancy. For many of the stars with the largest differences between the Hipparcos and FK5 proper motions, information on their duplicity was found in the literature. We thus suspected that the influence of astrometric binaries to be responsible for the discrepancy.

\section{Mean and instantaneous proper motions}

With its high measurement accuracy of better than 1 mas for the bright FK5 stars, Hipparcos was able to determine stellar motions with a precision of 1 mas/yr from observations during less than four years. A star's motion within this short time interval may, in the case of a double star, be completely different from the motion of its center of mass (Fig. 1). The observations used to construct the FK5, on the other hand, are less precise by a factor of about one hundred and in most cases they can therefore not resolve the wavy motion of a double star. The FK5 proper motions, derived from observations over about 250 years, reflect the star's mean motion in that time interval, and it will therefore be near to the motion of the center of mass, except for double stars with very long orbital periods. We believe that this basic difference in the time interval used - yielding 'instantaneous' Hipparcos and 'mean' FK5 proper motions - is responsible for the unexpectedly high dispersion of the differences between the Hipparcos and FK5 proper motions.

\section{Comparison between Hipparcos and FK5 proper motions}

We have computed the differences between the proper motions in the Hipparcos and the FK5 catalogue. In the following we have eliminated all stars for which we could find any information on suspected duplicity or for which Hipparcos itself has found indications of a non-linear motion. A subsample of 1202 stars was left from the 1535 FK5 stars. The FK5 proper motions were put onto the Hipparcos system by the following procedure. First some stars with highly discordant differences were eliminated, then the global rotation between both proper motion systems were determined and eliminated, and finally the remaining regional systematic differences were determined 


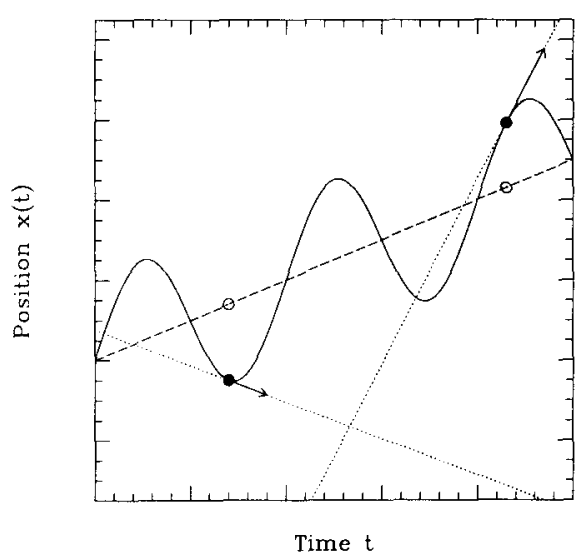

Figure 1. Wavy motion of the photo-center of an astrometric binary (solid curve) around the linear motion of its center of mass (dashed line). Two instantaneous positions and proper motions are indicated (filled dots; arrows). The linear predictions based on the instantaneous values are shown as dotted lines.

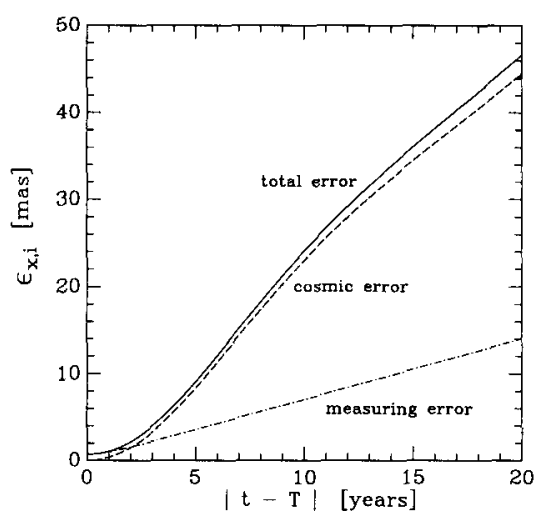

Figure 2. Mean error $\epsilon_{x, p}$ of a predicted instantaneous position $x_{p}(t)$ at an epoch $t$, based on a linear extrapolation using an instantaneous position and an instantaneaus proper motion observed at epoch $T$ (e.g. the Hipparcos epoch). The total mean error (solid) is the combination of the cosmic error (dashed) and the measuring error (dashed dotted).

and eliminated from the FK5 proper motions with the aid of the analytical method described by Bien et al. (1978). An iteration of this process has yielded no additional systematics.

For the the dispersion $D_{\mu, o b s}$ of the residual differences Hipparcos minus FK5 we obtained (averaged over $\mu_{\alpha} \cos \delta$ and $\mu_{\delta}$ ) :

$D_{\mu, o b s}=2.38 \mathrm{mas} / \mathrm{yr}$.

The error estimates given in the two catalogues yield expected mean errors (again averaged over both coordinates) of 0.77 mas/yr for the FK5, and 0.68 mas $/ y r$ for the Hipparcos proper motions. Taking also into account the average mean error $0.28 \mathrm{mas} / \mathrm{yr}$ of the derived systematic corrections we compute the total expected mean proper motion error as

$D_{\mu, e x p}=1.07$ mas $/ y r$.

Thus, the real dispersion of the observed differences exceeds the dispersion expected from the estimated proper motion accuracy in the two catalogues by a value $D_{\mu, \cos }$ which is

$$
D_{\mu, c o s}=\left(D_{\mu, o b s}^{2}-D_{\mu, \text { exp }}^{2}\right)^{\frac{1}{2}}=2.13 \mathrm{mas} / \mathrm{yr}
$$

This is about three times the errors estimated in the Hipparcos or the FK5. We explain this discrepancy with the presence of unresolved astrometric binaries in our sample. Hipparcos has measured for many of them 'instantaneous' proper motions which may deviate significantly from the mean FK5 proper motions (Fig. 1). We denoted this contribution in the observed dispersion as 'cosmic error'. This is not a real error, it merely results from our insufficient knowledge of a possible binaries.

\section{Supporting arguments for the presence of cosmic errors}

If our hypothesis is true that unresolved binaries produce the unexplained contribution in the observed dispersion, then the effect should depend on the star distance such that it decreases with increasing distance since the orbital displacement, measured in angular measure, becomes smaller. This effect is indeed significantly present as shown in Table 1. In various distance intervals are give the observed dispersion (resulting from comparing the published proper motions), the expected dispersion (resulting from the error estimates in the catalogues and including the uncertainty of the system) and the cosmic error (quadratic difference of both entries).

Another argument for the presence of the cosmic errors in the Hipparcos proper motions is given in Table 2. For the 1202 'apparently' single stars and the 95 so-called $g$ and $\dot{g}$ stars (stars for which significant deviations from linear motion were detected from the Hipparcos reduction procedure) we give the cosmic errors in proper motion (first line) and in position at the mean 
TABLE 1. Cosmic errors in proper motion as a function of the parallax

\begin{tabular}{crrrrr} 
& \multicolumn{5}{c}{ Parallax p [mas] } \\
& all & $\geq 20$ & $20-10$ & $10-5$ & $<5$ \\
\hline$D_{\mu, \text { obs }}$ & 2.38 & 2.94 & 2.50 & 2.05 & 1.58 \\
$D_{\mu, \text { exp }}$ & 1.07 & 1.01 & 1.06 & 1.11 & 1.11 \\
$D_{\mu, \text { cos }}$ & 2.13 & 2.76 & 2.26 & 1.72 & 1.12 \\
\hline
\end{tabular}

TABLE 2. Cosmic errors in proper motion and position at the Hipparcos and FK5 epoch, for the 'single' and the $g, \dot{g}$ stars

\begin{tabular}{rrr}
\hline 'type' of star & $\begin{array}{r}\text { 'single' } \\
1202\end{array}$ & $\begin{array}{r}\mathrm{g}, \dot{\mathrm{g}} \\
95\end{array}$ \\
\hline number of stars & 2.13 & 9.24 \\
$D_{\mu, \cos }[$ mas $/ y r]$ & 77.5 & 388.2 \\
$D_{p o s, \cos }\left(T_{F K 5}\right)[\mathrm{mas}]$ & $\mathbf{4 5 . 4}$ & 59.8 \\
$D_{\text {pos,cos }}\left(T_{H i p}\right)[\mathrm{mas}]$ & \\
\hline
\end{tabular}

FK5 epoch (about 1950, last but one line) and the mean Hipparcos epoch (about 1991.25, last line). Since the cosmic errors are dramatically larger for the $g$ and $\dot{g}$ stars, where Hipparcos had already found strong indication for orbital motion, we may conclude that unresloved binaries are also responsible for the cosmic error in our 'single' star sample. Comparing the dispersions in position at the FK5 and Hipparcos epoch makes obvious that the cosmic errors are primarily in the Hipparcos proper motions because their contribution over approximately 40 years epoch difference produces a significantly larger dispersion at the FK5 epoch than in the case of the FK5 proper motions which contribute to the dispersion at the Hipparcos epoch.

\section{Mathematical treatment of the cosmic errors}

The correct treatment of the cosmic errors makes use of correlation functions which describe the statistical correlation between the orbital displacements in position and motion relative to the mean motion. Since it is far beyond the scope of this brief presentation we refer the reader to Wielen (1997) for an introduction to this new field of 'statistical astrometry'. The correlation functions have been used by Wielen et al. (1997) to estimate the cosmic errors in a predicted Hipparcos position as a function of the time (Fig. 2). It is obvious that the cosmic errors dominate over the measuring errors, except for epochs very near to the mean Hipparcos epoch.

\section{Conclusions for the Reference Frame}

From 1998 onwards the International Celestial Reference Frame in optical wave lengths will be represented by the positions and motions of the stars in the Hipparcos catalogue. Since the orbital elements of the unresolved binaries in that catalogue, which are responsible for the cosmic errors described above, are distributed randomly in space and time, the cosmic errors will not introduce systematic errors into the frame. One has, however, to be aware that the precision of the positions and, in particular, of the proper motions, may be inferior to the estimates given in the catalogue. Since the cosmic errors dominate the error budget (except near the mean Hipparcos epoch) we suggest the use of stars at preferentially larger distances from the Sun for the maintenance of the reference frame, because the cosmic errors are less important for the distant stars (see Table 1).

\section{References}

Bien, R., Fricke, W., Lederle, T. \& Schwan, H., (1978) Methods for the comparison of systems of star positions to be applied in the construction of the FK5, Veroeff. Astron. Rechen-Inst., Heidelberg, No. 29, Verlag G. Braun, Karlsruhe

ESA, 1997, The Hipparcos Catalogue, ESA SP-1200

Fricke, W., Schwan, H., Lederle, T. et al., (1988) Fifth Fundamental Catalogue (FK5), Part I; Veroeff. Astron. Rechen-Inst., Heidelberg, No. 32, Verlag G. Braun, Karlsruhe

Wielen, R., (1997) Principles of statistical astrometry A\&A, 325, 367-382

Wielen, R., Schwan, H., Dettbarn, C., Jahreiss, H. \& Lenhardt, H. (1997) Statistical astrometry based on a comparison of individual proper motions and positions of stars in the FK5 and in the Hipparcos catalogue in HipparcosVenice' 97, ESA SP-402, 727-732 